\title{
Hidrograma de projeto em função da metodologia utilizada na obtenção da precipitação
}

\author{
Rita de C. F. Damé', Claudia F. A. Teixeira ${ }^{1}$, Viviane S. S. Terra ${ }^{2}$ \& José L. C. Rosskoff ${ }^{1}$
}

\begin{abstract}
RESUMO
Os dados de precipitação diária obtidos de pluviômetros, são mais facilmente encontrados que os pluviográficos, constituindo uma limitação uma vez que, em aplicações hidrológicas, como a transformação chuva-vazão, necessita-se de dados de precipitação de curtas durações. A solução desse problema vem sendo buscada ao longo do tempo através do desenvolvimento de modelos de desagregação de precipitação diária. O objetivo do trabalho é comparar os hidrogramas de projeto resultantes dos hietogramas estimados pelas relações Intensidade-Duração-Frequência (IDF), obtidos por dados pluviométricos e pluviográficos, para a localidade de Pelotas, RS. Para a desagregação da precipitação diária utilizou-se o Método das Relações, enquanto para a lâmina escoada e sua propagação em uma área de 7 km², a metodologia proposta foi a SCS (1972). Os resultados obtidos permitiram concluir que os valores de vazão de pico apresentaram uma diferença de 12,6, -4,4, 21,8, 38,7, 54,3 e 58,2\%, para os períodos de retorno de 2, 5, 10, 20, 50 e 100 anos, respectivamente, quando se utiliza a relação IDF gerada pelo método da desagregação de precipitação diária em relação à equação analítica.
\end{abstract}

Palavras-chave: hietograma, método das relações, intensidade-duração-frequência, vazão máxima de projeto

\section{Runoff hydrogram resulting from the methodology employed to record precipitation}

\begin{abstract}
Data of daily precipitation recorded by pluviometers are more easily found than those from pluviographic data. This often constitutes a limitation for hydrological applications such as the rainfall-runoff transformation, which needs to feed on short duration precipitation data. To overcome this constraint, the development of disaggregation models for daily precipitation has been sought for a long time. The aim of this study was to compare different runoff hydrograms resulting from hyetogram estimates from Intensity-Duration-Frequency (IDF) relationship between pluviometric and pluviographic data for the location of Pelotas, RS-Brazil. The relationship method was used to calculate daily precipitation disaggregation, while the runoff depth and its propagation were over a $7 \mathrm{~km}^{2}$ area and were determined through the methodology proposed by SCS (1972). The findings lead to the conclusion that the peak runoff values showed a difference of 12.6, -4.4, 21.8, $38.7,54.3$ and $58.2 \%$, for return periods of 2, 5, 10, 20, 50 and 100 years, respectively, when calculated through the IDF ratio, as compared to the analytical equation.
\end{abstract}

Key words: hyetogram, relationship method, intensity-duration-frequency, peak runoff flow 


\section{INTRODUÇÃO}

Em pequenas bacias rurais os dados de vazão observados são parcamente disponíveis ou inexistentes; portanto, para a obtenção da vazão máxima de projeto utilizada no dimensionamento de obras hidráulicas, retratando a segurança da obra, o custo envolvido na sua construção e, em consequência, a boa aplicação dos recursos públicos, é necessário utilizar modelos precipitação-vazão.

Neste processo, existem várias incertezas associadas, em que as principais são: (a) a variabilidade da distribuição temporal e espacial da precipitação máxima; (b) os parâmetros do modelo selecionado e (c) a estrutura do modelo hidrológico que simplifica os processos reais, introduzindo erros na representação do escoamento (Santos et al., 2001).

No que diz respeito à obtenção da precipitação máxima associada a um período de retorno, esta pode ser obtida mediante às relações intensidade-duração-frequência (IDF) de ocorrência da precipitação (Silva et al., 2002; 2003), tanto a partir de registros pluviográficos como pluviométricos.

McPherson (1958) questionou o uso das relações IDF obtidas a partir de registros pluviográficos, pelo fato das intensidades representarem a média de determinada duração, e não a história real do evento de precipitação, o que alteraria os resultados e avaliações dessas relações. A principal crítica se baseia em que a duração do evento utilizada na determinação dessas relações não é a duração real do evento e, sim, uma pequena parte da mesma, com duração maior.

Os dados de precipitação diária obtidos de pluviômetros, são mais facilmente encontrados que os pluviográficos, constituindo uma limitação uma vez que, em algumas aplicações hidrológicas, como a modelagem do processo chuva-vazão, infiltração, dinâmica de pântanos e obtenção das relações IDF, se necessitam de dados de precipitação em curtas durações, como 5-10-15-30 min e 1 h (Ferreira et al., 2005).

A solução deste problema vem sendo procurada ao longo do tempo através do desenvolvimento de modelos de desagregação de precipitação diária que estimam, a partir desta, o valor da precipitação com duração (t) e período de retorno (Tr) (Damé et al., 2006; Oliveira et al., 2008).

As relações IDF são usadas na estimativa do hietograma de projeto, definido como sequência de precipitações capazes de provocar a cheia de projeto (Fragoso Júnior, 2004). Alguns dos métodos para a estimativa do hietograma de projeto, baseados nas relações IDF, são o método de Chicago (Keifer \& Chu, 1957), o método proposto por Pilgrim \& Cordery (1975), o método do hietograma triangular proposto por Gamiz (2001) e o método dos blocos alternados (Bemfica et al., 2000).

Independentemente do método de estimativa do hietograma de projeto, a forma como é feita a distribuição temporal das alturas precipitadas não está relacionada com o fenômeno físico, ocorrendo apenas o rearranjo das alturas precipitadas, de forma a caracterizar a condição mais crítica.

A metodologia a ser utilizada para a distribuição temporal da precipitação exerce influência na determinação do escoamento superficial, ou seja, no volume e pico do hidrograma de projeto, os quais determinam o dimensionamento de obras hidráulicas.
Devido às incertezas inerentes a cada metodologia de estimativa do hietograma de projeto e, consequentemente, no hidrograma resultante, torna-se oportuno verificar se há ganho de informação nos hidrogramas estimados, utilizando-se os hietogramas obtidos mediante as relações IDF, a partir de dados pluviométricos, comparativamente aos estimados via registros pluviográficos.

Neste sentido, o objetivo do presente trabalho é comparar os hidrogramas de projeto resultantes dos hietogramas estimados pelas relações IDF de precipitação obtidos por dados pluviométricos e pluviográficos, para a localidade de Pelotas, RS.

\section{MATERIAL E MÉTODOS}

Utilizaram-se os dados de precipitação da localidade de Pelotas, RS, obtidos na Estação Agroclimatológica de Pelotas - Convênio EMBRAPA/UFPel, INMET. O posto selecionado localiza-se na latitude $31^{\circ} 51^{\prime}$, longitude $52^{\circ} 21^{\prime}$, a 13,2 m de altitude e o período de dados utilizados de 1982 a 1998, em função de que há disponibilidade de dados pluviométricos e pluviográficos.

Após a seleção do posto de precipitação foi constituída, a partir do software PROCEDA - Processamento de Dados Agroclimatológicos - (Viegas Filho et al., 2004), a série de precipitação máxima diária anual. A partir da série constituída, ajustaram-se os parâmetros da distribuição de Gumbel utilizando-se o software WINSTAT (Machado \& Conceição, 2006), para os períodos de retorno de 2, 5, 10, 20, 50 e 100 anos, e após plotados os valores ajustados e observados no papel de probabilidade deste modelo verificando, assim, a adequabilidade do ajuste.

Com o objetivo de obter a relação IDF a partir de registros pluviométricos, aplicou-se o Método das Relações (DAEE-CETESB, 1979; Oliveira et al., 2005) para desagregar a chuva diária; obtiveram-se, inicialmente, para a localidade de Pelotas, RS, os coeficientes correspondentes aos valores de lâmina precipitada, entre as diversas durações (15, $30,60 \mathrm{~min}$ e $1,6,12$ e $24 \mathrm{~h}$ ) e variados períodos de retorno (2, 5, 10, 20, 50 e 100 anos) para o que se utilizaram as leituras dos registros pluviográficos, nas mesmas durações e as séries ajustadas à distribuição de Gumbel, nos períodos de retorno estudados (Silveira, 2000).

Obtiveram-se, desta forma, os coeficientes de desagregação da precipitação diária nas durações 24 h/1 dia, 12 h/24 h, $6 \mathrm{~h} / 24 \mathrm{~h}, 1 \mathrm{~h} / 24 \mathrm{~h}, 30 \mathrm{~min} / 1 \mathrm{~h}$ e $15 \mathrm{~min} / 30 \mathrm{~min}$ e, por consequência, as alturas de lâminas, resultando na obtenção das relações IDF a partir de dados pluviométricos (Tabela 1).

Empregou-se a relação IDF do município de Pelotas, RS, obtida por Goulart et al. (1992), cuja expressão é:

$$
I=\frac{\left(1253,0975+64,7169 * \ln T_{r}\right)}{(t+5)^{0,8277^{*} T_{r}^{-0,0180}}}
$$

sendo I a intensidade máxima média de precipitação $\left(\mathrm{mm} \mathrm{h}^{-1}\right)$, Tr o período de retorno (anos) e t o tempo de duração da chuva (min). 
Tabela 1. Coeficientes de desagregação de dados pluviométricos

\begin{tabular}{lcccccc}
\hline \multirow{2}{*}{ Relações } & $\mathbf{7}$ Tr (anos) \\
\cline { 2 - 7 } $24 \mathrm{~h}$ /1 dia** & $\mathbf{2}$ & $\mathbf{5}$ & $\mathbf{1 0}$ & $\mathbf{2 0}$ & $\mathbf{5 0}$ & $\mathbf{1 0 0}$ \\
$12 \mathrm{~h} / 24 \mathrm{~h}$ & 0,90 & 0,96 & 0,97 & 0,98 & 0,99 & 0,99 \\
$6 \mathrm{~h} / 24 \mathrm{~h}$ & 0,79 & 0,83 & 0,85 & 0,86 & 0,87 & 0,88 \\
$1 \mathrm{~h} / 24 \mathrm{~h}$ & 0,47 & 0,47 & 0,48 & 0,48 & 0,48 & 0,49 \\
$30 \mathrm{~min} / 1 \mathrm{~h}$ & 0,76 & 0,72 & 0,70 & 0,68 & 0,67 & 0,66 \\
$15 \mathrm{~min} / 30$ min & 0,71 & 0,70 & 0,70 & 0,70 & 0,70 & 0,70 \\
\hline * maior valor de precipitação correspondente a um período consecutivo de 24 h; \\
** valor compreendido entre os horários de observação de precipitação pluvial
\end{tabular}

O hietograma de projeto foi obtido a partir do método dos blocos alternados (Marcellini, 1994), cujas etapas foram: (a) seleção da duração total da precipitação (td) e do intervalo de discretização $(\Delta t)$; (b) obtenção da intensidade de precipitação para cada duração, a partir da relação IDF, obtida por Goulart et al. (1992) e por desagregação de precipitação diária (dados pluviométricos); (c) transformação das intensidades em alturas de precipitação e acumuladas até o último intervalo de tempo; (d) cálculo dos incrementos dos totais acumulados por intervalo; (e) rearranjo dos incrementos ou blocos, obtidos em uma sequência tal que, no centro da duração da precipitação, se situe o bloco maior e, em seguida, os demais.

Para se obter o hietograma de projeto efetivo lançou-se mão do método do Soil Conservation Service (SCS, 1972; Singh et al., 2008), o qual considera a relação entre o armazenamento máximo, S (mm); a precipitação máxima acumulada, P (mm); a precipitação efetiva acumulada, Pe (mm); a quantidade armazenada no instante (P-Pe); as abstrações iniciais, $I_{a}$, (0,2 x S) e o parâmetro curva número, CN; segundo a metodologia utilizada, a precipitação efetiva foi obtida pelas seguintes relações:

$$
\begin{gathered}
P_{e}=\frac{\left(P-I_{a}\right)^{2}}{P-I_{a}+S} \\
S=\frac{25400}{C N}-254
\end{gathered}
$$

Obteve-se o hietograma efetivo da seguinte forma: (a) obtenção da lâmina efetiva para cada duração (Eqs. 2 e 3) e (b) cálculo dos incrementos da lâmina efetiva acumulada, por intervalo de tempo.

Para obtenção do hidrograma de projeto primeiro se estimaram os parâmetros do hidrograma unitário sintético triangular do SCS (1972), cuja metodologia consistiu, inicialmente, da estimativa do Hidrograma Unitário Curvilíneo da área em estudo, em que se adotou um valor de duração da chuva, $t_{r},\left(0,1333 \times t_{c}\right)$; calculou-se o tempo entre metade da precipitação e o instante de pico $\left(t_{p}\right)$, pela expressão, $t_{p}=0,6 t_{c}$; o tempo de instante do pico $\left(T_{p}\right)$ pela expressão, $T_{p}=\left(t_{r} / 2\right)+t_{p} ; o$ tempo entre o início e o fim do escoamento superficial, ou seja, o tempo de base $\left(\mathrm{t}_{\mathrm{b}}\right)$, $t_{\mathrm{b}}=2,67 \times\left(\left(\mathrm{t}_{\mathrm{r}} / 2\right)+\mathrm{t}_{\mathrm{p}}\right)$; a vazão de pico $\left(\mathrm{Q}_{\mathrm{p}}\right)$ pela expressão, $Q_{p}=(0,208 \times A) / T_{p}$, donde A é a área de drenagem expressa em $\mathrm{km}^{2}$ e $\mathrm{T}_{\mathrm{p}}$ o tempo de instante de pico em horas.

Uma área hipotética de $7 \mathrm{~km}^{2}$ foi utilizada, com com- primento de rio principal de $6 \mathrm{~km}$ e $117 \mathrm{~m}$ de desnível, cujo tempo de concentração da área foi de 72,2 min, estimado pela equação de Kirpch; para o parâmetro curva número, adotou-se um valor de 65, em função das características de tipo e uso do solo da área do entorno da estação agroclimatológica.

A partir dos valores obtidos de $T_{p}$ e $Q_{p}$, obtiveram-se as ordenadas do Hidrograma Unitário da Área, através do Hidrograma Unitário Adimensional (SCS, 1972). Para a estimativa do hidrograma de escoamento superficial foram utilizadas as ordenadas do hidrograma unitário e os valores de precipitação excedentes obtidos a partir dos hietogramas efetivos, estimados a partir das relações IDF, obtidas pela equação analítica apresentada por Goulart et al. (1992), e por desagregação de precipitação diária (dados pluviométricos).

Para avaliar o ganho de informação em termos de hidrograma resultante compararam-se, por meio de regressão linear e desvios relativos médios, os valores de vazão com período de retorno de 2, 5, 10, 20, 50 e 100 anos, obtidos a partir dos hietogramas de projeto, e efetivo estimados pelas relações IDF via registros pluviométricos em relação aos pluviográficos.

\section{RESULTADOS E DISCUSSÃO}

Apresentam-se, na Figura 1, os hietogramas de projeto e efetivo, para os períodos de retorno 2, 5, 10, 20, 50 e 100 anos, considerando-se que a relação IDF foi obtida a partir da equação analítica apresentada por Goulart et al. (1992) e a precipitação excedente obtida mediante metodologia do SCS (1972), cujo CN adotado foi 65, oriundo das características do tipo e uso do solo da bacia hipotética utilizada.

Observa-se que os valores totais escoados aumentam em função do período de retorno. Os percentuais de lâmina escoada em relação ao total precipitado foram de 4,5, 6,5, 8,1, 9,7, 12,1 e 14,0\%, para os períodos de retorno 2, 5, 10, 20, 50 e 100 anos, respectivamente, resultado este coerente com a estrutura da equação que representa a curva IDF (Eq. 1), visto que as intensidades máximas médias de precipitação, para a mesma duração, são diretamente proporcionais ao acréscimo do período de retorno.

A Figura 2 apresenta os hietogramas de projeto e efetivo, quando a relação IDF foi obtida mediante o método das relações (DAEE-CETESB, 1979) e a precipitação excedente mediante o SCS (1972). Comparando-se o hietograma de projeto com o efetivo, vê-se que os valores totais escoados aumentam em função do período de retorno e os percentuais de lâmina escoada em relação ao total precipitado foram de 2,0, 6,3, 9,4, 12,3, 16,1 e 18,7\%, para os períodos de retorno 2, 5, 10, 20, 50 e 100 anos, respectivamente.

Apesar dos valores dos coeficientes de correlação (Figura 3), obtidos entre as chuvas de projeto estimadas pela equação IDF (Goulart et al., 1992) e por desagregação de chuva diária (DAEE-CETESB, 1979) terem sido superiores a 0,90, para todos os períodos de retorno, os valores de precipitação obtidos pelo método da desagregação de precipitação diária se encontram superestimados para os períodos de retorno de 


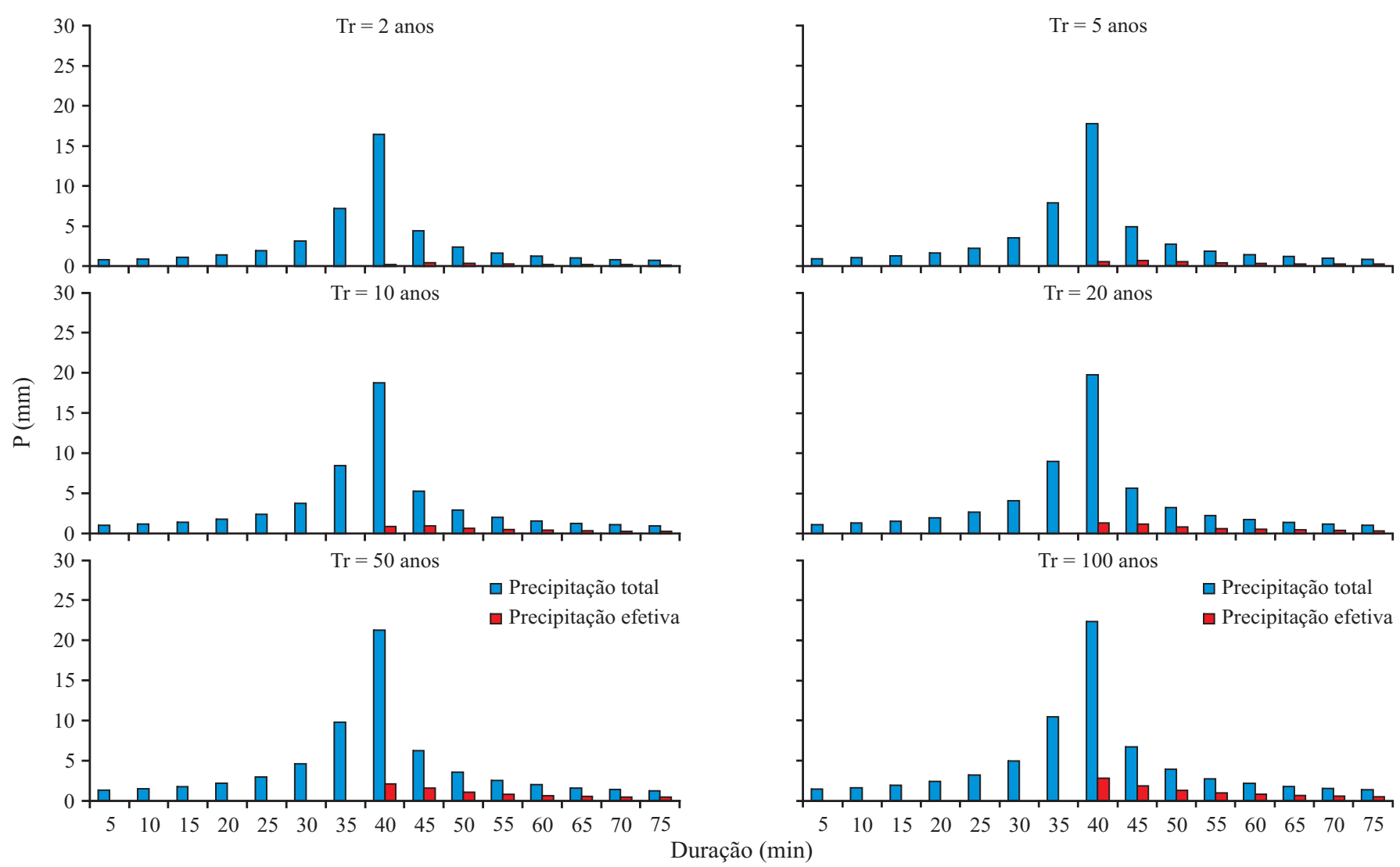

Figura 1. Hietogramas de projeto e efetivo, para os períodos de retorno 2, 5, 10, 20, 50 e 100 anos, considerando-se a relação IDF obtida a partir da equação analítica (Goulart et al., 1992)
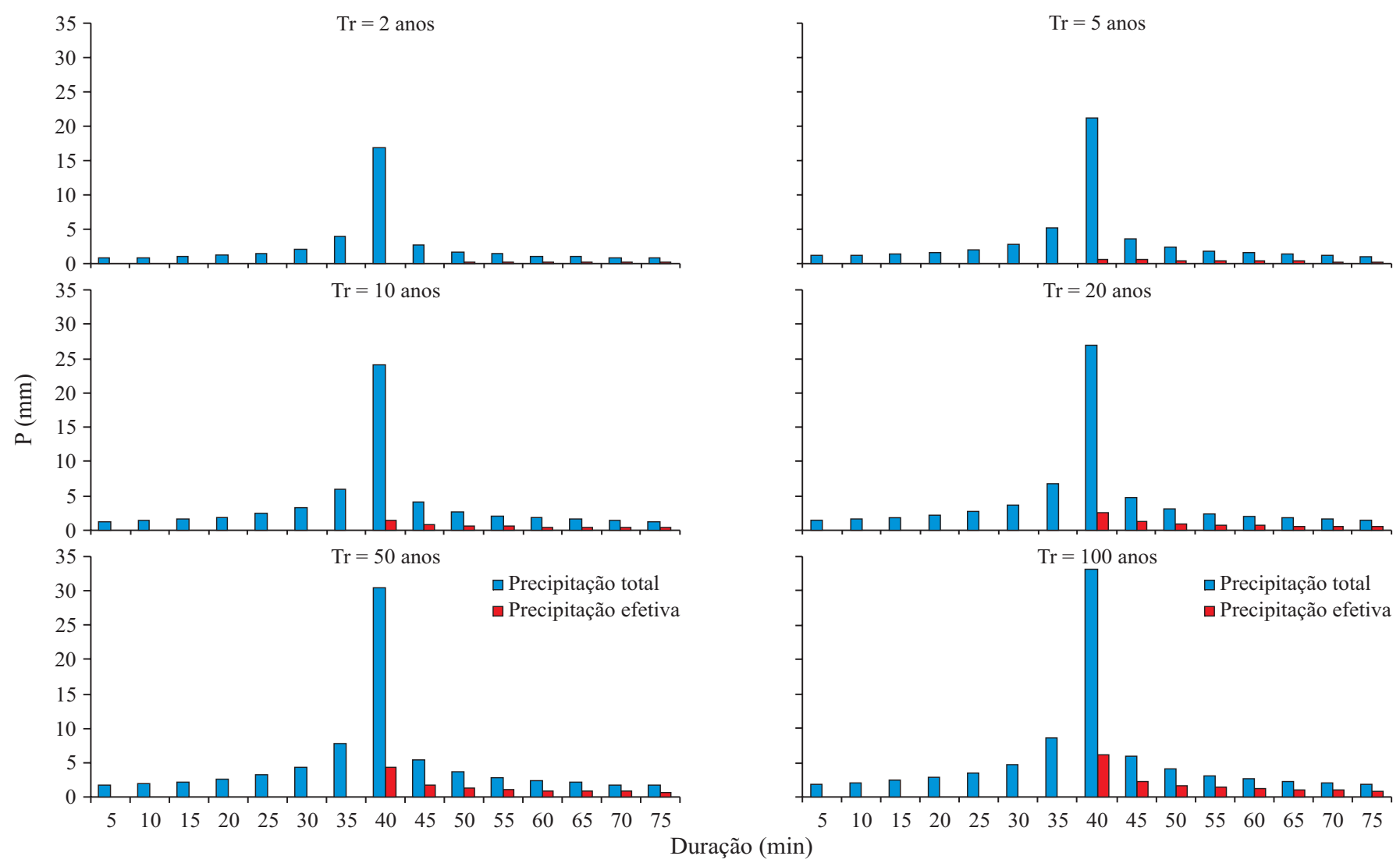

Figura 2. Hietogramas de projeto e efetivo para os períodos de retorno 2, 5, 10, 20, 50 e 100 anos, quando a relação intensidade-duração-frequencia (IDF) foi obtida mediante o método das relações (DAEE-CETESB, 1979) 

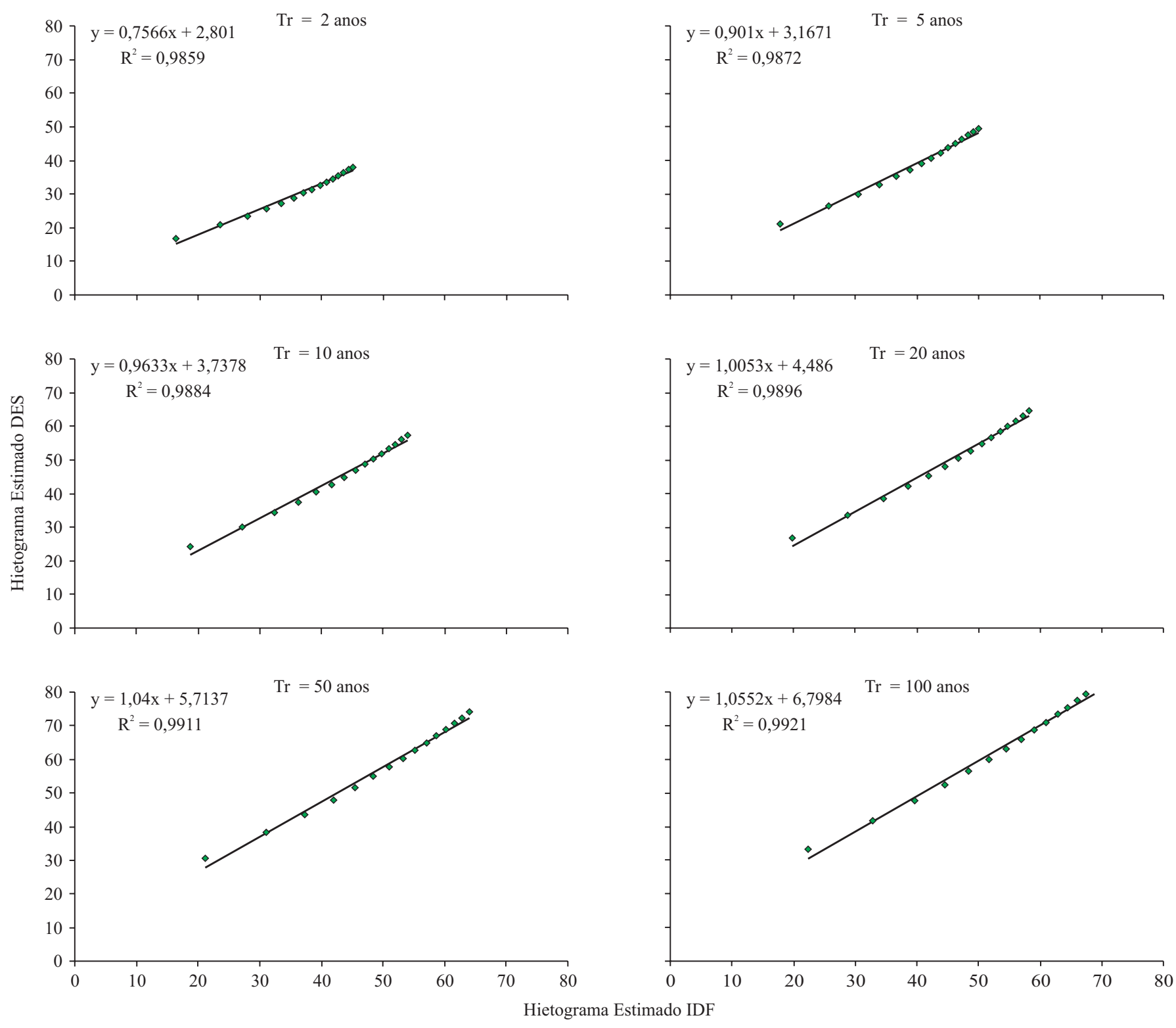

Figura 3. Comparação entre os hietogramas de projeto estimados por desagregação de precipitação diária e mediante a equação analítica de Goulart et al. (1992), bem como as respectivas equações lineares, para cada período de retorno

10, 20, 50 e 100 anos, nos respectivos valores percentuais $5,1,10,4,15,4$ e 18,2\% (Figura 4).

Oliveira et al. (2000) empregaram a metodologia de desagregação da precipitação diária (DAEE-CETESB, 1979) para obter as relações IDF para diferentes períodos de retorno, em algumas localidades no estado de Goiás e as compararam com as obtidas por Costa \& Brito (1999), para a mesma região. Os autores concluíram que a metodologia se mostrou adequada, uma vez que os valores de erro relativo médio foram inferiores a $14,4 \%$.

Para o período de retorno de 2 anos, os valores de chuva de projeto se apresentaram subestimados em 16,6\%; já para 5 anos, foram praticamente coincidentes para ambas as metodologias utilizadas, apresentando diferença percentual de $1,9 \%$.

Segundo Moreti et al. (2003) e Damé et al. (2005), na seleção do período de retorno, para elaboração de projetos de drenagem de superfície, deve-se considerar os custos da obra, o grau de risco, a vida útil da obra, o tipo de estrutura e a facilidade de reparo e ampliação da mesma. Para projetos hidroagrícolas, Pruski (1993) recomenda um período de retorno 10 anos. No presente trabalho a diferença percentual foi de apenas 5,1\%, para período de retorno de 10 anos, valor inferior ao encontrado por Oliveira et al. (2000), verificando a adequabilidade da metodologia de desagregação de precipitação diária para estimativa do hietograma de projeto.

Em relação aos resultados de vazão de projeto pode-se observar, na Figura 5, uma variação significativa na vazão de pico, de acordo com o método utilizado para a estimativa do hietograma de projeto.

Observando-se as Figuras 1, 2 e 5, percebe-se haver coerência entre os valores dos hietogramas efetivos e hidrogramas de projeto resultantes, para todos os períodos de retorno estudados. Os hietogramas obtidos mediante desagregação 

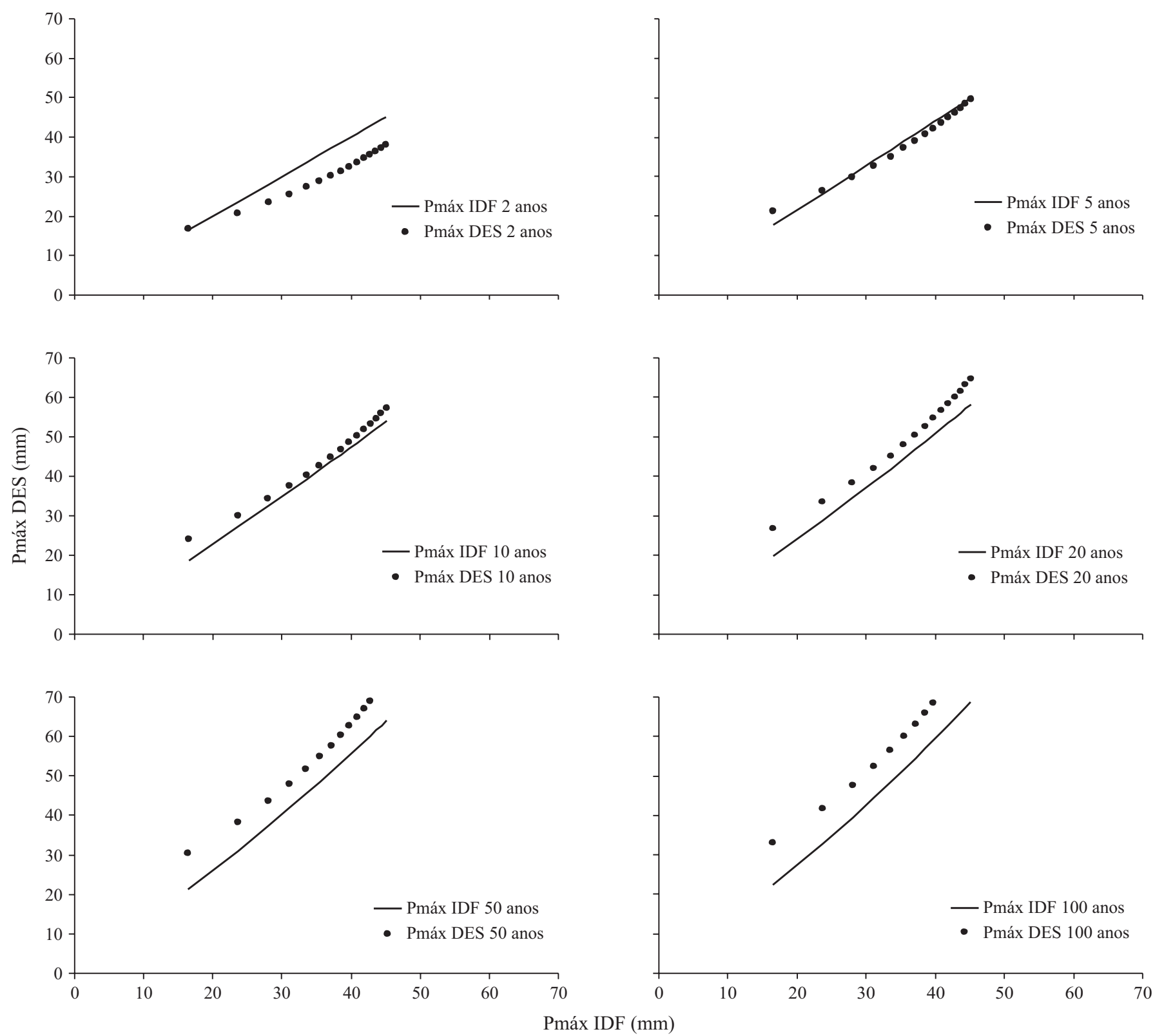

Figura 4. Comparação entre as alturas precipitadas estimadas por desagregação de precipitação diária e mediante a equação analítica de Goulart et al. (1992), para cada período de retorno

de precipitação diária se apresentaram superestimados em relação aos obtidos, conforme a curva IDF de Goulart et al. (1992), nos períodos de retorno 10, 20, 50 e 100 anos, assim como os hidrogramas resultantes (Figura 5).

A análise em separado dos valores de vazão de pico (Tabela 2) mostrou uma diferença de 12,6, -4,4, 21,8, 38,7, 54,3 e 58,2\%, para os períodos de retorno de 2, 5, 10, 20, 50 e 100 anos, respectivamente, quando se utiliza a relação IDF gerada pelo método da desagregação de precipitação diária. Segundo Marcellini (1994) o período de retorno exerce papel fundamental na determinação da vazão de projeto; o autor constatou, também, que para uma mesma duração da chuva de projeto a variação da vazão entre os períodos de retorno de 10 a 50 anos foi da ordem de 42,0\%; para as demais durações da chuva de projeto esta mesma diferença também foi observada.
Tabela 2. Vazões de pico estimadas a partir dos hietogramas de projeto obtidos por desagregação de precipitação diária (Q DES) e equação analítica de Goulart et al. (1992) (Q IDF), para todos os períodos de retorno estudados

\begin{tabular}{lcccrcc}
\hline Tr (anos) & $\mathbf{2}$ & $\mathbf{5}$ & $\mathbf{1 0}$ & $\mathbf{2 0}$ & $\mathbf{5 0}$ & $\mathbf{1 0 0}$ \\
Q DES $\left(\mathrm{m}^{3} \mathrm{~s}^{-1}\right)$ & 0,98 & 4,79 & 8,32 & 13,02 & 19,39 & 24,09 \\
Q IDF $\left(\mathrm{m}^{3} \mathrm{~s}^{-1}\right)$ & 0,87 & 5,01 & 6,83 & 9,39 & 12,57 & 15,23 \\
\hline
\end{tabular}

No trabalho de Oliveira et al. (2005) os valores da vazão de pico e o volume de água escoada superficialmente, para o período de retorno de 10 anos, apresentaram uma diferença de 4,0\% na vazão de pico do escoamento superficial, quando utilizaram a relação IDF gerada pelo método da desagregação de chuva, para o município de Santa Terezinha de Goiás; para o município de Itumbiara, a vazão de pico do 

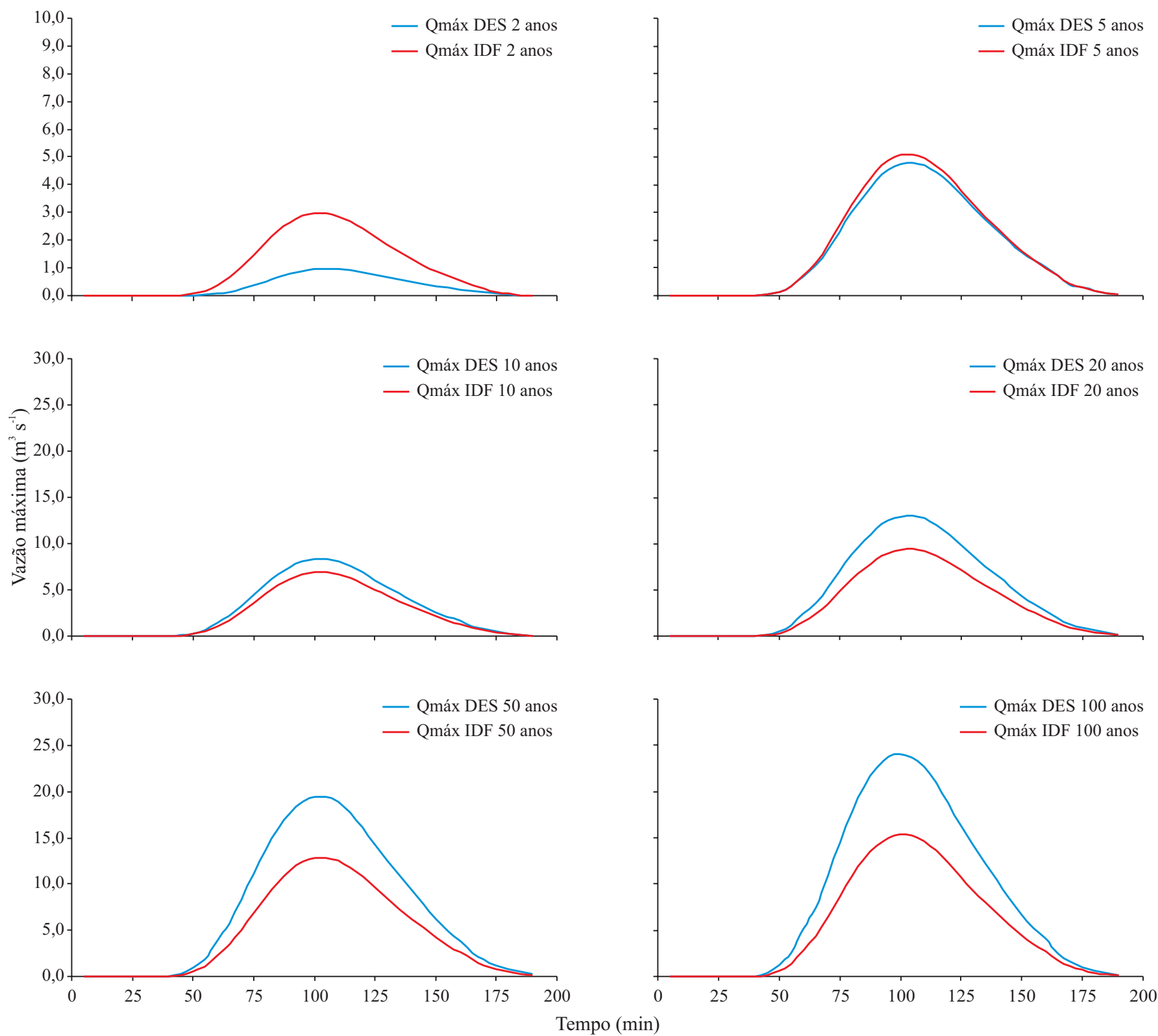

Figura 5. Comparação entre os hidrogramas de projeto estimados a partir dos hietogramas de projeto estimados por desagregação de precipitação diária e equação analítica de Goulart et al. (1992), para cada período de retorno

escoamento superficial foi $78,0 \%$ menor quando empregaram a mesma metodologia o que, segundo os autores, poderá vir a comprometer o dimensionamento das obras de terraceamento.

Resultados desta natureza permitem verificar que o hidrograma de projeto depende, concomitantemente, de diversas variáveis, como a precipitação, uso e tipo de solo, capacidade de infiltração do mesmo, tempo de resposta da bacia hidrográfica para uma mesma entrada de chuva, dentre outros, e como a metodologia empregada na estimativa da chuva de projeto é apenas uma das variáveis envolvidas e vem imbuída de questionamentos, desde McPherson (1958), o qual ponderou sobre o uso das relações IDF obtidas a partir de registros pluviográficos, que representam a média de chuva de determinada duração, e não a história real do evento, o que altera os resultados e avaliações dessas relações.
Outra ponderação a ser feita se refere ao modelo de escoamento superficial utilizado (SCS, 1972) que considera, em sua estrutura, a precipitação e as características do solo quanto à sua condição hidrológica, ou seja, o binômio infiltração-escoamento superficial. Neste método, o parâmetro CN (curva número) é o que representa a capacidade de infiltração do solo da bacia hidrográfica, caracterizada pela umidade antecedente do solo, o tipo do solo e a ocupação do mesmo.

Morel-Seytoux (1987) faz algumas críticas ao método, o qual diverge da teoria física da infiltração em que, para um solo saturado, a taxa de infiltração decresce com o tempo, independentemente da chuva. O autor observa que o método do SCS (1972) produz uma curva decrescente da infiltração somente para uma taxa de chuva constante, e ainda aponta falhas na estimativa das abstrações iniciais referentes à 
estimativa da taxa de interceptação e ao armazenamento nas depressões do solo.

No presente trabalho, como o valor do parâmetro CN foi fixado (65), não houve interferência deste nos resultados obtidos do hidrograma de projeto e assim foi possível avaliar apenas a influência da metodologia de estimativa da relação IDF sobre a vazão resultante.

Apesar dos desvios encontrados com o uso da estimativa da chuva de projeto mediante o uso da desagregação da chuva diária, esta é uma alternativa possível para a obtenção do hidrograma de projeto naquelas bacias desprovidas de equações IDF, obtidas a partir de registros pluviográficos. Ainda há de se considerar que, muitas vezes, as equações IDF disponíveis não apresentam o período de dados pluviográficos que foi utilizado na sua obtenção, bem como as restrições quanto à duração e período de retorno; assim, a desagregação de chuva diária pode retratar a realidade hidrológica da bacia hidrográfica em estudo, com maior proximidade de uma relação IDF, em que não são conhecidos os limites de sua aplicação.

\section{CONCLUSÕES}

1. Para o período de retorno de 10 anos, recomendado para projetos hidroagrícolas, os valores de precipitação obtidos pelo método da desagregação de precipitação diária se encontram superestimados em 5,1\%.

2. Os percentuais de lâmina escoada em relação ao total precipitado foram 9,4 e 8,1\%, para o período de retorno de 10 anos, quando o hietograma efetivo foi obtido via registros pluviográficos e pluviométricos, respectivamente.

3. Os valores de vazão de pico apresentaram diferença de $12,6,-4,4,21,8,38,7,54,3$ e 58,2\%, para os períodos de retorno de 2, 5, 10, 20, 50 e 100 anos, respectivamente, quando se utilizou a relação IDF gerada pelo método da desagregação de precipitação diária em relação à equação analítica.

\section{LITERATURA CITADA}

Bemfica, D. C.; Goldenfum, J. A.; Silveira, A. L. L. Análise da aplicabilidade de padrões de chuva de projeto de Porto Alegre. Revista Brasileira de Recursos Hídricos, v.5, n.4, p.5-16, 2000.

Costa, A. R.; Brito, V. F. Equações de chuva intensa para Goiás e sul de Tocantins. In: Simpósio Brasileiro de Recursos Hídricos, 13, Belo Horizonte. Resumos... Belo Horizonte: ABRH, 1999. CD Rom

DAEE-CETESB-Departamento de Água e Energia Elétrica Companhia de Tecnologia de Saneamento Ambiental. Drenagem urbana: Manual de projeto. São Paulo: DAEE-CETESB. 1979. 476p.
Damé, R. C. F.; Pedrotti, C. B. M.; Cardoso, M. A. G.; Silveira, C. P.; Duarte, L. A. D.; Ávila, M. S. V; Moreira, A. C. Comparação entre curvas intensidade-duração-frequência de ocorrência de precipitação obtidas a partir de dados pluviográficos com àquelas estimadas por técnicas de desagregação de chuva diária. Revista Brasileira de Agrociência, v.12, n.4, p.505-509, 2006.

Damé, R. C. F.; Teixeira, C. F. A.; Moura C.; Machado, R.; Beskow, S. Análise do impacto de um evento de precipitação ocorrido na cidade de Pelotas/RS. Revista de Ciências AgroAmbientais, v.3, p.15-25, 2005.

Ferreira, J. C.; Daniel, L. A.; Tomazela, M. Parâmetros para equações mensais de estimativas de precipitação de intensidade máxima para o estado de São Paulo - Fase I. Ciência e Agrotecnologia, v.29, n.6, p.1175-1187, 2005.

Fragoso Júnior, C. R. Regionalização da vazão máxima instantânea com base na precipitação de projeto. Revista Eletrônica de Recursos Hídricos, v.1, n.1, p.5-13, 2004.

Gamiz, M. C. Determinación de la tormenta de diseño. Ingeniería Hidráulica y Ambiental, v.22, n.2, p.27-30, 2001.

Goulart, J. P.; Maestrini, A. P.; Neibel, A. L. Relação intensidade-duração-frequência de chuvas em Pelotas, RS. Revista Brasileira de Meteorologia, v.7, n.1, p.543-552, 1992.

Keifer, C. J.; Chu, H. H. Synthetic storm pattern for drainage design. Journal of the Hydraulics Division, ASCE, v.83, n.4, Ser. HY, p.1-25, Proc. Paper 1332, 1957.

Machado, A. A.; Conceição, A. R. WinStat: Sistema de análise estatística para windows. Universidade Federal de Pelotas. 2006. htttp://www.ufpel.edu.br/ amachado. 26 Jan. 2008.

Marcellini, S. S. Análise de critérios para a determinação das tormentas de projeto e sua influência nos hidrogramas em pequenas bacias hidrográficas. São Paulo: USP, 1994. 176p. Dissertação Mestrado

McPherson, M. B. Discussion of synthetic storm pattern for drainage design. Journal of the Hydraulics Division, v.84, n.1, p.49-60, 1958.

Morel-Seytoux, H. J. Multiphase flow in porous media. In: Developments in Hydraulic Engineering. London: Pavel Novak, Elsevier Applied Science Publishers, 1987. p.103-174.

Moreti, D.; Carvalho, M. P.; Mannigel, A. R.; Medeiros, L. R. Importantes características de chuva para a conservação do solo e da água no município de São Manuel (SP). Revista Brasileira de Ciência do Solo, v.27, p.713-725, 2003.

Oliveira, L. F. C.; Antonini, J. C. A.; Griebeler, N.P. Estimativas de chuvas intensas para o Estado de Goiás. Engenharia Agrícola, v.28, n.1, p.22-33, 2008.

Oliveira, L. F. C.; Cortês, F. C.; Barbosa, F. O. A.; Romãos, P. A.; Carvalho, D. F. Estimativa das equações de chuvas intensas para algumas localidades no estado de Goiás pelo método da desagregação de chuvas. Pesquisa Agropecuária Tropical, v.30, n.1, p.23-27, 2000.

Oliveira, L. F. C.; Cortês, F. C.; Wehr, T. R.; Borges, L. B.; Sarmento, P. H. L.; Griebeler, N. P. Intensidade-duração-frequência de chuvas intensas para localidades no estado de Goiás e Distrito Federal. Pesquisa Agropecuária Tropical, v.30, n.1, p.13-18, 2005. 
Pilgrim, D. H.; Cordery, I. Rainfall temporal patterns for design floods. Journal of the Hydraulics Division, v.101, n.1, p.81-95, 1975.

Pruski, F. F. Desenvolvimento de metodologia para dimensionamento de canais de terraços. Viçosa: UFV, 1993. 97p. Tese Doutorado

Santos, R. S.; Tucci, C.; Silveira, A. L. L.; Meneses Filho, A. S. Estimativa do hidrograma de projeto com base na incerteza dos parâmetros do modelo. Revista Brasileira de Recursos Hídricos, v.6, n.1, p.29-41, 2001.

SCS - Soil Conservation Service. Hydrology. In: National engineering handbook. Washington: USDA, 1972. p.101-1023.

Silva, D. D.; Gomes Filho, R. R.; Pruski, F. F.; Pereira, S. B.; Novaes, L. F. Chuvas intensas no Estado da Bahia. Revista Brasileira de Engenharia Agrícola e Ambiental, v.6, n.2, p.362-367, 2002.
Silva, D. D.; Pereira, S. B.; Pruski, F. F.; Gomes Filho, R. R.; Lana, A. M. Q.; Baena, L. G. N. Equações de intensidadeduração-frequência da precipitação pluvial para o Estado de Tocantins. Engenharia na Agricultura, v.11, n.1-4, p.7-14, 2003.

Silveira, A. L. L. Equação para os coeficientes de desagregação de chuva. Revista Brasileira de Recursos Hídricos, v.5, n.4, p.143-147, 2000.

Singh, P. K.; Bhunya, P. K.; Mishra, S. K.; Chaube, U. C. A sediment graph model based on SCS-CN method. Journal of Hydrology, v.349, p.244-255, 2008.

Viegas Filho, J. S.; Conceição, A. R.; Machado, A. A.; Lanna, A. E. L.; Damé, R. C. F. PROCEDA - Processador de dados do SAGBAH, Pelotas, 2004. 